\title{
LA CONFERENCIA DE EL CAIRO Y LA AFIRMACIÓN DE LOS DERECHOS SEXUALES Y REPRODUCTIVOS, COMO BASE PARA LA SALUD SEXUAL Y REPRODUCTIVA
}

\author{
THE CAIRO CONFERENCE AND THE ASSERTION OF SEXUAL AND REPRODUCTIVE \\ RIGHTS AS A BASIS FOR SEXUAL AND REPRODUCTIVE HEALTH
}

\author{
Susana Galdos Silva1,2,a
}

\author{
Movimiento Manuela Ramos. Lima, Perú. \\ Universidad Peruana Cayetano Heredia. Lima, Perú. \\ Licenciada en educación, máster en salud pública, sexualidad y ciencias, MPH in Sexuality and Family Science
}

Recibido: 03-07-13; Aprobado: 21-08-13

\begin{abstract}
RESUMEN
El artículo se enfoca en la Conferencia Internacional sobre Población y Desarrollo de El Cairo, llevada a cabo en Egipto en 1994, en la cual se trataron temas referidos a derechos sexuales y reproductivos, a las acciones para mejorar la situación de las niñas, el estatus de la mujer, la situación de los adolescentes y la igualdad de género, como componentes básicos para mejorar la salud sexual y reproductiva de la población. Las recomendaciones producto de esta conferencia conforman el programa de acción. En el presente documento se desarrollan, además, algunos temas que durante la conferencia generaron largas discusiones para llegar a un consenso. Resume el seguimiento al programa de acción en los años posteriores y se detiene en el informe del gobierno peruano y la sociedad civil hacia los veinte años de la conferencia.
\end{abstract}

Palabras clave: Conferencias de salud; Salud sexual y reproductiva; Derechos sexuales y reproductivos (fuente: DeCS BIREME)

\begin{abstract}
The article focuses on the International Conference on Population and Development held in El Cairo, Egypt, in 1994. The Conference addressed issues related to sexual and reproductive rights, actions to be adopted to improve the situation of young girls, the status of women, the situation of adolescents and gender equality as basic components to improve the sexual and reproductive health of the population. The concluding recommendations in this conference constitute the action program. This document also addresses some issues that generated long discussions to reach consensus during the conference. It summarizes the follow up performed on the action program in the following years and ends with the report of the Peruvian government and the civil society twenty years after the conference.
\end{abstract}

Key words: Health conferences; Sexual and reproductive health; Sexual and reproductive rights (source: MeSH NLM).

\section{INTRODUCCIÓN}

En la Conferencia Internacional sobre la Población y el Desarrollo de El Cairo de 1994 se rompe con el esquema de las conferencias anteriores de priorizar las preguntas de cuántos somos y dónde estamos. Las conferencias anteriores sobre población, Bucarest 1974 y México 1984, centraban la discusión en el tema de la población mundial y los procesos migratorios. El temor a la sobrepoblación y a la escasez de recursos promovió el control de la fecundidad como política que facilitaría el desarrollo de los países. La Conferencia de El Cairo centra el debate en el desarrollo de los grupos sociales y de los sujetos individuales como eje de la dinámica de población. Reconoce que si bien hombres y mujeres tienen realidades y necesidades comunes, las instituciones sociales perpetúan una posición de desventaja de las mujeres con relación a los hombres. Es por ello que en la conferencia se realza el empoderamiento de la mujer y la mejora de la situación de las niñas, conjuntamente con el derecho a la salud sexual y reproductiva, como estrategia importante para la reducción de la pobreza, mejora de la salud y la calidad de vida. Bien se podría decir que el programa de acción (PA) que se discutió y acordó en la Conferencia de El Cairo, asumió lo acordado en conferencias previas, como el Programa 21 y la Declaración de Rio de 1992 de 
la aprobado en la Conferencia de las Naciones Unidas sobre el Medioambiente y el Desarrollo; los acuerdos de la Cumbre Mundial a favor de la Infancia en 1990, y la Conferencia Mundial de Derechos Humanos de Viena de 1993.

Asimismo, otros insumos importantes fueron las recomendaciones formuladas en las cinco conferencias regionales sobre población, diversas reuniones preparatorias subregionales, documentos preparados por organizaciones de la sociedad civil, así como los informes especiales de más de 140 países.

\section{LA CONFERENCIA}

Representantes de 179 estados, con diversos niveles de desarrollo económico-social, regímenes de gobierno, creencias religiosas, culturas, valores, costumbres, roles asignados a hombres y mujeres, ideas sobre la familia, y la sexualidad discutieron el PA que debía ser aprobado por consenso. Los grupos organizados de mujeres, a los que la Organización de Naciones Unidas (ONU) abrió las puertas para participar con los delegados de los gobiernos, marcaron la diferencia en los debates. En el caso peruano, ya desde 1980 los grupos feministas tenían como eje de lucha el derecho de las mujeres a la salud y controlar su reproducción, la disminución de la mortalidad materna y sus causas, incluido el acceso al aborto y a una vida libre de violencia y con igualdad de oportunidades. La conferencia de El Cairo fue una oportunidad para que los representantes de la sociedad civil, y activistas de los derechos de las mujeres y de la salud sexual y reproductiva expusieran sus argumentos y debatieran con los representantes de los gobiernos, a fin de lograr consensos que mantuvieran el espíritu de los profundos cambios en el enfoque de la población y el desarrollo que el PA tiene. En total 10757 participantes fueron registrados. Además, 4200 representantes de más de 1500 organizaciones no gubernamentales, de 133 países asistieron al Foro de Organizaciones no Gubernamentales, reunión independiente que se celebró al mismo tiempo que la Conferencia.

\section{EL PROGRAMA DE ACCIÓN}

El PA es un conjunto de recomendaciones con las cuales se comprometieron los Estados y cuya aplicación sería enmarcada en el derecho soberano de cada país. Respecto a él, Nafis Sadik, secretaria general de la Conferencia Internacional sobre Población y Desarrollo de El Cairo y directora ejecutiva del Fondo de Población de las Naciones Unidas, afirmó: /... Este programa de acción tiene capacidad potencial para cambiar el mundo.
EI PA fue concebido como un conjunto de metas cualitativas y cuantitativas encaminadas a lograr los objetivos en materia de población y desarrollo. Abarcó los siguientes capítulos: relaciones entre la población; el crecimiento económico y el desarrollo sostenible; la igualdad y equidad entre los sexos y la habilitación de la mujer; la familia, sus funciones, derechos, composición y estructura; crecimiento y estructura de la población; derechos reproductivos y salud reproductiva; salud, morbilidad y mortalidad; distribución de la población, urbanización y migración interna; migración internacional; población, desarrollo y educación; tecnología, investigación y desarrollo; actividades nacionales; cooperación internacional; colaboración con el sector no gubernamental, y actividades complementarias a la conferencia. Cada artículo comprende: bases para la acción, objetivos y medidas (1).

\section{DEBATES Y NUDOS CRIITICOS DE LA CONFERENCIA}

En algunos artículos del PA, los debates consumieron horas hasta encontrar términos aceptables por todos los países que, al mismo tiempo, fueran claros en el objetivo. Así, algunos capítulos y ciertos términos generaron grandes debates. Entre el 5 y 13 de septiembre se trataron los capítulos referidos a principios, derechos reproductivos y salud reproductiva, salud mortalidad y morbilidad (donde se discutió el aborto) y unificación de la familia en inmigración internacional; estos capítulos demandaron gran tiempo, ya que hubo una gran dificultad para lograr consenso y algunos países manifestaron reservas, a pesar de que en los principios se establece claramente que la implementación del PA se enmarca en el derecho y las leyes de cada país.

En particular, el capítulo sobre derechos reproductivos y salud reproductiva fue largamente discutido. La idea inicial fue la inclusión del respeto a los derechos sexuales y derechos reproductivos de las personas en el PA. El término "derechos sexuales" no fue aceptado pero si se aceptaron los "derechos reproductivos". Este rechazo se basó en la negación de la diversidad sexual y en el temor que al aceptar derechos sexuales se estuviera también aceptando y reconociendo el derecho de las personas a definir su propia identidad. De manera similar, la salud sexual motivó grandes debates que finalmente lograron consenso al aceptar la definición de la Organización Mundial de la Salud referida a salud sexual e incorporándola como parte de la salud reproductiva aun cuando la lógica indica que la salud reproductiva es parte de la salud sexual.

En ese mismo capítulo, la aceptación de que toda persona tiene el derecho a decidir libre y responsablemente el 
número de hijos que quiere tener fue un triunfo frente a posiciones que afirmaban que debería ser la pareja, hombre y mujer, quienes de común acuerdo debían decidir el número de hijos y los métodos para planificar su familia. Finalmente, el párrafo se redactó indicando que los derechos reproductivos se basan en el reconocimiento del derecho básico de todas las parejas y todas las personas. Ese mismo párrafo menciona que los derechos reproductivos incluyen el derecho a disfrutar del nivel más elevado posible de salud sexual y salud reproductiva.

El debate más largo se centró en el aborto y la necesidad de despenalizarlo o legalizarlo a fin de eliminar los abortos en condiciones de riesgo causantes de alta mortalidad materna. Finalmente, se redactó un texto que enfatiza no promover el aborto como método de planificación familiar, atender los efectos en la salud de la mujer que producen los abortos realizados en condiciones no adecuadas y ofrecer información y servicios a fin de prevenir abortos.

No fue posible tampoco incluir una visión más amplia y reconocimiento a los diversos tipos de familia. Los textos alternativos especificaban familias, en plural, pero el consenso optó por reforzar la idea de un solo tipo de familia, en singular aunque se hace mención a que hay diversos tipos de familias.

La posibilidad de que los adolescentes recurran a servicios de consejería y de atención a su salud, sin la presencia o permiso de los padres fue otro tema de discusión. La redacción final junta el derecho y responsabilidad de los padres de proporcionar orientación en materia de sexualidad, y exhorta a los países a que los adolescentes tengan acceso a información y servicios apropiados.

\section{IMPORTANTES DEFINICIONES}

En la conferencia, además, quedaron sentadas algunas definiciones importantes ${ }^{(1)}$. La salud reproductiva es un estado general de bienestar físico, mental y social, y no de mera ausencia de enfermedades o dolencias, en todos los aspectos relacionados con el sistema reproductivo y sus funciones y procesos. En consecuencia, la salud reproductiva entraña la capacidad de disfrutar de una vida sexual satisfactoria y sin riesgos y de procrear, y la libertad para decidir hacerlo o no hacerlo, cuándo y con qué frecuencia. Esta última condición lleva implícito el derecho del hombre y la mujer a obtener información y de planificación de la familia de su elección, así como a otros métodos para la regulación de la fecundidad que no estén legalmente prohibidos, y acceso a métodos seguros, eficaces, asequibles y aceptables, el derecho a recibir servicios adecuados de atención de la salud que permitan los embarazos y los partos sin riesgos, y otorguen a las parejas las máximas posibilidades de tener hijos sanos. En consonancia con esta definición de salud reproductiva, la atención de la salud reproductiva se define como el conjunto de métodos, técnicas y servicios que contribuyen a la salud y al bienestar reproductivo al evitar y resolver los problemas relacionados con la salud reproductiva. Incluye también la salud sexual, cuyo objetivo es el desarrollo de la vida y de las relaciones personales y no meramente el asesoramiento y la atención en materia de reproducción y de enfermedades de transmisión sexual.

Teniendo en cuenta la definición que antecede, los derechos reproductivos abarcan ciertos derechos humanos que ya están reconocidos en las leyes nacionales, en los documentos internacionales sobre derechos humanos y en otros documentos pertinentes de la ONU aprobados por consenso. Esos derechos se basan en el reconocimiento del derecho básico de todas las parejas e individuos a decidir libre y responsablemente el número de hijos, el espaciamiento de los nacimientos y el intervalo entre estos, así como a disponer de la información y de los medios para ello, y el derecho a alcanzar el nivel más elevado de salud sexual y reproductiva. También incluye su derecho a adoptar decisiones relativas a la reproducción sin sufrir discriminación, coacciones ni violencia, de conformidad con lo establecido en los documentos de derechos humanos. En ejercicio de este derecho, las parejas y los individuos deben tener en cuenta las necesidades de sus hijos nacidos y futuros, además de sus obligaciones con la comunidad. La promoción del ejercicio responsable de esos derechos de todos debe ser la base primordial de las políticas y programas estatales y comunitarios en la esfera de la salud reproductiva, incluida la planificación de la familia. Como parte de este compromiso, se debe prestar plena atención a la promoción de relaciones de respeto mutuo e igualdad entre hombres y mujeres, y particularmente a las necesidades de los adolescentes en materia de enseñanza y de servicios con el objetivo de que puedan asumir su sexualidad de modo positivo y responsable. La salud reproductiva está fuera del alcance de muchas personas de todo el mundo a causa de factores como: los conocimientos insuficientes sobre la sexualidad humana, y la información y los servicios insuficientes o de mala calidad en materia de salud reproductiva; la prevalencia de comportamientos sexuales de alto riesgo; las prácticas sociales discriminatorias; las actitudes negativas hacia las mujeres y las niñas, y el limitado poder de decisión que tienen muchas mujeres respecto de su vida sexual y reproductiva. En la mayoría 
de los países los adolescentes son particularmente vulnerables a causa de su falta de información y de acceso a los servicios pertinentes. Las mujeres y los hombres de más edad tienen problemas especiales en materia de salud reproductiva, que no suelen encararse de manera adecuada.

Si bien no fue posible lograr la aprobación de los textos que reafirmaban claramente los derechos sexuales y reproductivos de la persona en general y de los adolescentes, en particular, a nivel mundial las organizaciones de la sociedad civil reconocen que el PA indica a los gobiernos el camino a seguir hacia la práctica de los derechos sexuales y reproductivos, los derechos de las mujeres y hacia el desarrollo humano con salud y bienestar. La aplicación del PA debe orientarse por la definición más amplia de salud reproductiva, que incluye la salud sexual (2).

\section{SEGUIMIENTO DEL PROGRAMA DE ACCIÓN, A 5, 10 Y 15 AÑOS DE LA CONFERENCIA}

El seguimiento al PAse ha dado en la mayoría de los países a nivel gubernamental y a través de las organizaciones e instituciones de la sociedad civil con el apoyo de las agencias de cooperación internacional, principalmente de la ONU. Encuentros nacionales y regionales han ido describiendo lo logrado y lo pendiente ${ }^{(3-6)}$.

En Perú, la Mesa Tripartita de Seguimiento al PA conformada en 1997 e impulsada por las organizaciones feministas, fue un espacio de trabajo conjunto y de coordinación de representantes de la sociedad civil y representantes del gobierno, en particular del Ministerio de Salud y los organismos internacionales como el Fondo de Población de las Naciones Unidas, con el objetivo de impulsar la implementación del PA, detectando vacíos, dificultades y experiencias exitosas. Ios indicadores seleccionados estaban relacionados con violencia sexual contra las niñas; responsabilidad masculina en sexualidad y reproducción; participación de las organizaciones de mujeres en instancias de decisión; acceso de los adolescentes a información y servicios de salud sexual y reproductiva; calidad de los servicios de salud sexual y reproductiva; atención humanizada del aborto inseguro, y prevención y atención integral a personas afectadas por el VIH/SIDA.

Este espacio de diálogo y concertación entre Estado y sociedad civil, que justamente daba cumplimiento a la recomendación del PA de coordinar acciones hacia el logro de objetivos, quedó trunco cuando el programa de planificación familiar del Ministerio de
Salud desarrolló esterilizaciones forzadas e impuso metas de esterilizaciones, principalmente en mujeres de poblaciones rurales e indígenas, usando los programas de planificación familiar como medio para reducir la pobreza. Esta violación de los derechos sexuales y reproductivos de las mujeres invalidó la posibilidad de continuar un trabajo conjunto en la medida que las acciones del Ministerio de Salud negaban lo esencial del PA: el derecho de cada persona a decidir sobre su reproducción. Evaluaciones posteriores han sido trabajadas a nivel nacional y regional, tanto por organizaciones de mujeres y de defensa de los derechos sexuales y reproductivos como por redes latinoamericanas; sin embargo, ya no fue posible un informe conjunto entre el Estado y la sociedad civil.

A nivel mundial el Fondo de Población de las Naciones Unidas en el documento "Seguimiento a Cairo, Medidas Clave, Cairo +5 Décimo aniversario del Programa de Acción" resume los principales acuerdos que los gobiernos consideran deben priorizarse ${ }^{(5)}$. Se debe medir el acceso universal a la salud sexual y reproductiva no solo por anticoncepción (cerrando la brecha de demanda insatisfecha) sino también por mortalidad materna, enfermedades de transmisión sexual y el VIH/SIDA. De igual forma, se debe reconocer la gravedad de la pandemia de VIH/SIDA y legislar para prevenir la discriminación, atender la transmisión horizontal y proveer medicamentos retrovirales. Se debe reiterar que el aborto no debe promoverse como método de planificación familiar y que debe atenderse en condiciones adecuadas donde la ley lo permita y ofrecer servicios de calidad para ocuparse de las complicaciones. Asimismo, se debe procurar que la comunidad internacional y el sector privado faciliten transferencias de tecnologías para que los países puedan producir, almacenar y distribuir anticonceptivos inocuos y eficaces.

En el mismo documento se reafirman los objetivos del PA y se incorporan nuevos elementos. Así, en lo referente al tema de igualdad, equidad y potenciación del papel de la mujer se pide a los gobiernos que garanticen la promoción y protección de los derecho humanos de las mujeres; que firmen, ratifiquen y apliquen la convención sobre la eliminación de todas las formas de discriminación contra la mujer y que integren una perspectiva de género en todos los procesos de formulación, y aplicación de políticas en la prestación de servicios, especialmente los de salud sexual y reproductiva.

En lo referido a los derechos de procreación y salud reproductiva, se puntualiza la prevención y tratamiento de las enfermedades de transmisión sexual, incluido el 
virus de inmunodeficiencia humana (VIH). Los Estados acordaron prestar atención específica a los jóvenes (de 15 a 24 años) para que para el año 2005, al menos el $90 \%$, y para el año 2010 , al menos el $95 \%$, tengan acceso a la información necesaria, y a la educación y servicios adecuados. Esto incluye los métodos preventivos tales como el condón de la mujer y del hombre, los exámenes voluntarios, la consejería, y el seguimiento. Las tasas de infección por el VIH entre los jóvenes debe ser un indicador de referencia, con la meta de asegurar que para el año 2005 la prevalencia entre este grupo sea reducida mundialmente, y en un $25 \%$ en los países más afectados, y que para el año 2010 la prevalencia en este grupo etario sea reducida mundialmente en un $25 \%{ }^{(5)}$.

El Centro Latinoamericano y Caribeño de Demografía (CELADE), División de Población de la Comisión Económica para América Latina y el Caribe (CEPAL), realizó una encuesta a fines del año 2000 para conocer si los países tenían indicadores de seguimiento al PA. Posteriormente, el Comité Especial sobre Población y Desarrollo de la CEPAL en la reunión de la Mesa Directiva en Santiago de Chile ${ }^{(4)}$, los países de la región reafirmaron su compromiso con los objetivos del plan de acción y con las medidas clave. La declaración de Santiago insta a los países de la región a implementar políticas económicas y sociales para reducir la pobreza y la desigualdad en el marco de la implementación del PA.

\section{HACIA LOS 20 AÑOS DE LA CONFERENCIA: EL INFORME PERUANO}

La encuesta mundial elaborada por el Fondo de Población de las Naciones Unidas sobre el cumplimiento del PA de la Conferencia Internacional sobre la Población y el Desarrollo y su seguimiento después del 2014, motivó que el Ministerio de la Mujer y Poblaciones Vulnerables elabore un informe sobre el cumplimiento de compromisos (2). Dicho informe involucró a la mayoría de ministerios y direcciones relacionados con los temas de salud y población, y registra que entre los años 2000 al 2010, se dieron 28 políticas al respecto, e informa específicamente sobre mortalidad materna, uso de anticonceptivos modernos y evolución de los casos notificados de infección por $\mathrm{VIH}$. Con relación a la igualdad de género, equidad y empoderamiento de las mujeres, este documento indica políticas en tres aspectos: políticas orientadas a promover las equidades de género; políticas contra la violencia de género, y políticas para incrementar la participación de las mujeres en los procesos políticos.
Lamentablemente, tanto en salud reproductiva como en igualdad de género, el documento no analiza las dificultades en la implementación de las políticas dadas, ni recoge demandas de los vacíos y problemas que la sociedad civil ha dado a conocer.

Finalmente, en dicho informe se señala que: Los temas pendientes de abordar y que necesitan una ampliación en el conocimiento a través de investigaciones para identificar políticas son: i) los vínculos entre la dinámica de la población y el ambiente, b) los procesos de migración interna y los patrones de urbanización, c) el proceso de envejecimiento, las condiciones de vida de las personas de la tercera edad, las relaciones intergeneracionales y d) los cambios en las familias.

Por otro lado, la Mesa de Vigilancia por los derechos sexuales y reproductivos, que agrupa 16 organizaciones de la sociedad civil, y con la participación de otras catorce instituciones, ha elaborado el documento "Cairo +20 , hacia una lectura de la plataforma para la acción de Cairo a 20 años en el Perú (1994-2014)" (2), donde analiza lo logrado y lo pendiente en los temas de: embarazo en adolescentes, mortalidad materna, aborto, acceso a métodos anticonceptivos, VIH/SIDA y educación sexual integral. Cada tema es presentado a partir de los objetivos del PA, el contexto nacional, las políticas dadas, el presupuesto asignado y finaliza con recomendaciones al Estado.

\section{REFLEXIONES FINALES}

Consideramos necesario hacer notar que el principal freno a la aceptación de temas referidos a derechos sexuales y reproductivos viene de sectores religiosos que en nuestro país influyen a nivel político y social. El Estado peruano que, oficialmente, es un Estado laico, no lo es en la práctica y se confunde el respeto a creencias religiosas, que corresponden al derecho a la libertad de culto de cada persona, con políticas de salud pública que deben estar basadas en evidencias científicas. Esta es una de las razones por las que quedan aún pendientes temas claves para la salud, que solo necesitan de voluntad política para ser atendidos, como son: reconocimiento de la diversidad sexual, aprobación del aborto en caso de violación, educación sexual integral y anticoncepción oral de emergencia en los servicios de salud.

Fuentes de financiamiento: autofinanciado.

Conflictos de interés: el autor declara no tener conflictos de interés. 


\section{REFERENCIAS BIBLIOGRÁFICAS}

1. United Nations Population Information Network (POPIN). Informe de la Conferencia Internacional sobre la Población y el Desarrollo (El Cairo, 5 a 13 de septiembre de 1994) [Internet]. New York: POPIN [citado el 12 de febrero de 2013]. Disponible en: http://www.un.org/popin/icpd/ conference/offspa/sconf13.html

2. Perú, Ministerio de la Mujer y Poblaciones Vulnerables (MIMP). Perú: Resultados de la encuesta mundial sobre el cumplimiento del programa de acción de la conferencia internacional en población y desarrollo y su seguimiento después de 2014. Lima: MIMP; 2013.

3. United Nations Population Fund (UNFPA). Medidas clave para seguir ejecutando el Programa de Acción de la Conferencia Internacional sobre la Población y el Desarrollo [Internet]. Lima: UNFPA; 2004 [citado el 12 de febrero de 2013]. Disponible en www. unfpa.org.pe/cipd/cipd5/

4. Fondo de Población de las Naciones Unidas. Comisión Económica para América Latina y El Caribe. Centro Latinoamericano y caribeño de demografía. Cinco años después de El Cairo. América Latina y El Caribe: logros y desafíos en la ejecución del programa de acción de la conferencia internacional sobre la población y el desarrollo. Santiago de Chile: UNFPA; 1999.

5. Fondo de Población de las Naciones Unidas. Cairo, Cairo +5 , Cairo +10 . Diez años Conferencia Internacional sobre Población y Desarrollo. Lima: UNFPA; 2004.
6. Conferencia Internacional sobre la Población y el Desarrollo. Cairo +20: hacia una lectura de la plataforma para la acción der Cairo a 20 años en el Perú (1994-2014). Lima: Mesa de Vigilancia por los Derechos Sexuales y Reproductivos, PROMSEX, Movimiento Manuela Ramos, CENDIPP; 2013

Correspondencia: Susana Galdos Silva Dirección: Av. Juan Pablo Fernandini 1550, Lima 21, Perú.

Teléfono: (511) 423-8840

Correo electrónico: galdos44@gmail.com

\section{Consulte la versión electrónica de la} Revista Peruana de Medicina Experimental y Salud Pública en www.pubmed.gov

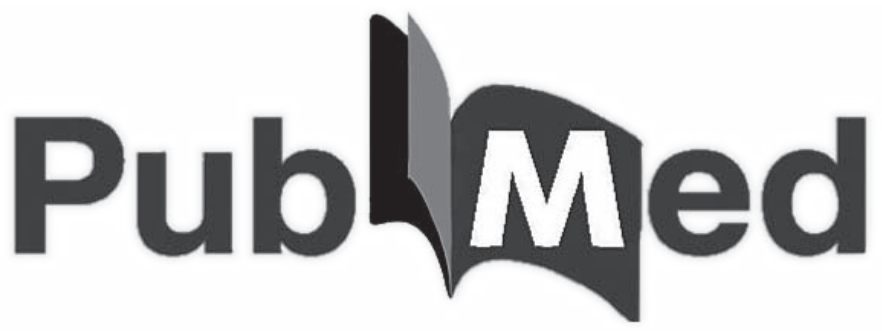

